\title{
REAL HYPERSURFACES OF A COMPLEX PROJECTIVE SPACE IN TERMS OF HOLOMORPHIC DISTRIBUTION
}

\author{
By
}

Sadahiro MAEDA and Seiichi UdAgAwA

\section{Introduction.}

Real hypersurfaces in a complex projective space have been studied by many differential geometers (for example, see [1], [2], [3], [7], [14] and [15]). In this paper, we study real hypersurfaces in $P_{n}(\boldsymbol{C})$ from the point of view of holomorphic distribution, where $P_{n}(\boldsymbol{C})$ denotes an $n$-dimensional complex projective space with Fubini-Study metric of constant holomorphic sectional curvature 4.

R. Takagi ([13]) showed that all homogeneous real hypersurfaces in $P_{n}(C)$ are realized as the tubes of constant radius over compact Hermitian symmetric spaces of rank 1 or 2 . Namely, he proved the following

THEOREM A ([13]). Let $M$ be a homogeneous real hypersurface of $P_{n}(\boldsymbol{C})$. Then $M$ is locally congruent to one of the following:

$\left(\mathrm{A}_{1}\right)$ a geodesic hypersphere (, that is, a tube over a hyperplane $P_{n-1}(\boldsymbol{C})$ ),

$\left(\mathrm{A}_{2}\right)$ a tube over a totally geodesic $P_{k}(C)(1 \leqq k \leqq n-2)$,

(B) a tube over a complex quadric $\boldsymbol{Q}_{n-1}$,

(C) a tube over $P_{1}(\boldsymbol{C}) \times P_{(n-1) / 2}(\boldsymbol{C})$ and $n(\geqq 5)$ is odd,

(D) a tube over a complex Grassmann $G_{2,5}(C)$ and $n=9$,

(E) a tube over a Hermitian symmetric space $S O(10) / U(5)$ and $n=15$.

On the other hand, Kimura $([4],[5])$ constructed a certain class of nonhomogeneous real hypersurfaces in $P_{n}(C)$, which are called ruled real hypersurfaces in $P_{n}(\boldsymbol{C})$.

Let $M$ be a real hypersurface of $P_{n}(\boldsymbol{C})$ and denote by $T M$ the tangent bundle of $M$. Set $\xi=-J N$, where $J$ is the complex structure tensor of $P_{n}(\boldsymbol{C})$ and $N$ is a local unit normal vector field of $M$ in $P_{n}(C)$. Then we may write as $T_{x} M=T_{x}^{0} M+\boldsymbol{R}\left\{\boldsymbol{\xi}_{x}\right\}$ at any fixed point $x$ of $M$, where $T_{x}^{0} M$ is a $J$-invariant subspace of $T_{x} M$. Let $A_{2}$ be the second fundamental form for the subbundle Received April 18, 1989. 
$T^{0} M$ in $T P_{n}(\boldsymbol{C})$ over $M$ (see $\S 3$ ), where $T P_{n}(\boldsymbol{C})$ is the tangent bundle of $P_{n}(\boldsymbol{C})$. Set $A^{0}=\left.A_{2}\right|_{T^{0} M}$. Then $A^{0}$ may be interpreted as a smooth section of $\operatorname{Hom}\left(T^{0} M\right.$, $\operatorname{Hom}\left(T^{0} M, N^{0} M\right)$ ), where $N^{0} M$ is the orthogonal complement of $T^{0} M$ in $T P_{n}(\boldsymbol{C})$ with respect to the metric on $T P_{n}(\boldsymbol{C})$, which is also a subbundle of $T P_{n}(\boldsymbol{C})$. Each of $T^{0} M$ and $N^{0} M$ has a connection induced from $T P_{n}(\boldsymbol{C})$ and hence $\operatorname{Hom}\left(T^{0} M, \operatorname{Hom}\left(T^{0} M, N^{0} M\right)\right)$ has a connection, which is denoted by $\nabla^{0}$ (cf. [6]).

In Section 3, we show the condition that $\nabla_{X}^{0} A^{0}=0$ for any $X \in T^{0} M$ implies that either $\xi$ is a principal curvature vector and the shape operator $A$ of $M$ in $P_{n}(\boldsymbol{C})$ is $\eta$-parallel or $T^{0} M$ is integrable, hence either $M$ is locally a homogeneous real hypersurface of type $A_{1}, A_{2}$ or $B$, or $M$ is foliated by complex hypersurface of $P_{n}(\boldsymbol{C})$ with parallel second fundamental form, which is $P_{n-1}(\boldsymbol{C})$ or a complex hyperquadric $\boldsymbol{Q}_{n-1}(\boldsymbol{C})$ by the well-known result of NakagawaTakagi $([10])$. Moreover, we determine real hypersurfaces $M$ 's (in $P_{n}(C)$ ) which satisfy the condition " $T^{0} M$ is a curvature invariant subspace of $T M$ and $\xi$ is not a principal curvature vector" by using Kimura's work [4].

In Section 2, we give some characterizations of homogeneous real hypersurfaces of type $A_{1}$ and $A_{2}$.

\section{Preliminaries.}

Let $M$ be a real hypersurface of $P_{n}(\boldsymbol{C})$. In a neighborhood of each point, we choose a unit normal vector field $N$ in $P_{n}(C)$. The Riemannian connections $\tilde{\nabla}$ in $P_{n}(C)$ and $\nabla$ in $M$ are related by the following formulas for arbitrary vector fields $X$ and $Y$ on $M$ :

$$
\begin{gathered}
\tilde{\nabla}_{X} Y=\nabla_{X} Y+g(A X, Y) N, \\
\tilde{\nabla}_{X} N=-A X,
\end{gathered}
$$

where $g$ denotes the Riemannian metric of $M$ induced from the Fubini-Study metric $G$ of $P_{n}(\boldsymbol{C})$ and $A$ is the shape operator of $M$ in $P_{n}(\boldsymbol{C})$. An eigenvector $X$ of the shape operator $A$ is called a principal curvature vector. Also an eigenvalue $\lambda$ of $A$ is called a principal curvature. In what follows, we denote by $V_{\lambda}$ the eigenspace of $A$ associated with eigenvalue $\lambda$. It is known that $M$ has an almost contact metric structure induced from the complex structure $J$ of $P_{n}(\boldsymbol{C})$, that is, we define a tensor field $\phi$ of type $(1,1)$, a vector field $\xi$ and a 1 -form $\eta$ on $M$ by $g(\phi X, Y)=G(J X, Y)$ and $g(\xi, X)=\eta(X)=G(J X, N)$. Then we have

$$
\phi^{2} X=-X+\eta(X) \xi, \quad g(\xi, \xi)=1, \quad \phi \xi=0 .
$$

From (1.1), we easily have 


$$
\begin{gathered}
\left(\nabla_{X} \phi\right) Y=\eta(Y) A X-g(A X, Y) \xi, \\
\nabla_{X} \xi=\phi A X .
\end{gathered}
$$

Let $\widetilde{R}$ and $R$ be the curvature tensors of $P_{n}(\boldsymbol{C})$ and $M$, respectively. Since the curvature tensor $\tilde{R}$ has a nice form, we have the following Gauss and Codazzi equations :

$$
\begin{aligned}
& g(R(X, Y) Z, W)= g(Y, Z) g(X, W)-g(X, Z) g(Y, W) \\
&+ g(\phi Y, Z) g(\phi X, W)-g(\phi X, Z) g(\phi Y, W) \\
&-2 g(\phi X, Y) g(\phi Z, W)+g(A Y, Z) g(A X, W) \\
&-g(A X, Z) g(A Y, W), \\
&\left(\nabla_{X} A\right) Y-\left(\nabla_{Y} A\right) X=\eta(X) \phi Y-\eta(Y) \phi X-2 g(\phi X, Y) \xi .
\end{aligned}
$$

It is well-known that there does not exist a real hypersurface $M$ of $P_{n}(\boldsymbol{C})$ satisfying $\nabla A=0$ (, that is, the second fundamental form of $M$ is parallel). Here we recall the following notion: The second fundamental form is called $\eta$ parallel if $g\left(\left(\nabla_{X} A\right) Y, Z\right)=0$ for any $X, Y$ and $Z$ which are orthogonal to $\xi$. We note that the second fundamental form of homogeneous real hypersurfaces of type $A_{1}, A_{2}, B$ and ruled real hypersurfaces is $\eta$-parallel (cf. Theorem 5). We say that $M$ is a ruled real hypersurface if there is a foliation of $M$ by complex hyperplanes $P_{n-1}(\boldsymbol{C})$. More precisely, let $T^{0} M$ be the distribution defined by $T_{x}^{0} M=\left\{X \in T_{x} M: X \perp \xi\right\}$ for $x \in M$. Then $T^{0} M$ is integrable and its integral manifold is a totally geodesic submanifold $P_{n-1}(\boldsymbol{C})$. In the following, we use the same terminology and notations as above unless otherwise stated. Now we prepare without proof the following in order to prove our Theorems:

Theorem B ([11], [12]). Let $M$ be a real hypersurface of $P_{n}(\boldsymbol{C})$. Then the following are equivalent:

(i) $M$ is locally congruent to one of homogeneous real hypersurfaces of type $A_{1}$ and $A_{2}$.

(ii) $L_{\xi} g=0$, where $L$ is the Lie derivative. Namely, $\xi$ is an infinitesimal isometry.

(iii) $\phi A=A \phi$.

THEOREM C ([5]). Let $M$ be a real hypersurface of $P_{n}(C)$. Then the second fundamental form of $M$ is $\eta$-parallel and $\xi$ is a principal curvature vector if and only if $M$ is locally congruent to one of homogeneous real hypersurfaces of type $A_{1}, A_{2}$ and $B$. 
THEOREM D ([5]). Let $M$ be a real hypersurface of $P_{n}(C)$. Then the second fundamental form of $M$ is $\eta$-parallel and the holomorphic distribution $T^{\circ} M(=$ $\{X \in T M: X \perp \xi\})$ is integrable if and only if $M$ is locally congruent to a ruled real hypersurface of $P_{n}(C)$.

PROPOSITION A ([9]). If $\xi$ is a principal curvature vector, then the corresponding principal curvature $\alpha$ is locally constant.

Proposition B ([9]). Assume that $\xi$ is a principal curvature vector and the corresponding principal curvature is $\alpha$. If $A X=r X$ for $X \perp \xi$, then we have $A \phi X$ $=((\alpha r+2) /(2 r-\alpha)) \phi X$.

Proposition C ([9]). Let $M$ be a real hypersurface of $P_{n}(C)$. Then the following are equivalent:

(i) $M$ is locally congruent to one of homogeneous ones of type $A_{1}$ and $A_{2}$.

(ii) $g\left(\left(\nabla_{X} A\right) Y, Z\right)=-\eta(Y) g(\phi X, Z)-\eta(Z) g(\phi X, Y)$ for any vector fields $X$, $Y$ and $Z$ on $M$.

Proposition D ([5]). Let $M$ be a real hypersurface of $P_{n}(C)$. Then the following are equivalent:

(i) The holomorphic distribution $T^{0} M=\{X \in T M: X \perp \xi\}$ is integrable.

(ii) $g((\phi A+A \phi) X, Y)=0$ for any $X, Y \in T^{0} M$.

\section{Homogeneous real hypersurfaces of type $A_{1}$ and $A_{2}$.}

In this section we provide some characterizations of homogeneous real hypersurfaces of type $A_{1}$ and $A_{2}$ in $P_{n}(C)$. Motivated by Theorem $\mathrm{B}$, first of all we prove the following

THEOREM 1. Let $M$ be a real hypersurface of $P_{n}(C)$. Then the following are equivalent:

(i) $M$ is locally congruent to one of homogeneous real hypersurfaces of type $A_{1}$ and $A_{2}$.

(ii) $L_{\xi} \phi=0$, that is, $\xi$ is an infinitesimal automorphism of $\phi$.

Proof. For any $X \in T M$, we have

$$
\begin{aligned}
\left(L_{\xi} \phi\right)(X) & =[\xi, \phi X]-\phi([\xi, X]) \\
& =\nabla_{\xi}(\phi X)-\nabla_{\phi X} \xi-\phi\left(\nabla_{\xi} X-\nabla_{X} \xi\right) \\
& =\left(\nabla_{\xi} \phi\right) X-\nabla_{\phi X} \xi+\phi\left(\nabla_{X} \xi\right)
\end{aligned}
$$




$$
\begin{aligned}
& =\eta(X) A \xi-g(A \xi, X) \xi-\phi A \phi X+\phi^{2} A X(\text { from (1.4) and (1.5)) } \\
& =\eta(X) A \xi-g(A \xi, X) \xi-\phi A \phi X-A X+\eta(A X) \xi(\text { from (1.3)) } \\
& =\eta(X) A \xi-\phi A \phi X-A X .
\end{aligned}
$$

Since $\left(L_{\xi} \phi\right)(\xi)=0$, the above calculation asserts that $L_{\xi} \phi=0$ is equivalent to

$$
A X=-\phi A \phi X \quad \text { for any } X(\perp \xi) \text {. }
$$

From (1.3) and (2.1) we find

$$
\phi A X=A \phi X-\eta(A \phi X) \xi \quad \text { for any } X(\perp \xi) .
$$

Then we see

$$
\begin{aligned}
\phi^{2} A X & =-A X+\eta(A X) \xi & (\text { from }(1.3)) & \\
& =\phi A \phi X & & (\text { from }(1.3) \text { and }(2.2)) \\
& =-A X & & (\text { from (2.1)) }
\end{aligned}
$$

that is, $\eta(A X)=0$ for any $X(\perp \xi)$ so that $\xi$ is a principal curvature vector. And hence, we get $\eta(A \phi X)=g(A \phi X, \xi)=g(\phi X, A \xi)=0$. Here we suppose that $L_{\xi} \phi=0$. Then from (2.2) we obtain $\phi A X=A \phi X$ for any $X(\perp \xi)$. Moreover, from the fact that $\xi$ is a principal curvature vector, it follows that $\phi A \xi=A \phi \xi(=0)$. Then " $L_{\xi} \phi=0$ " implies " $\phi A=A \phi$ ". On the other hand " $\phi A=A \phi$ " yields the equation (2.1), that is, " $L_{\xi} \phi=0$ ". Therefore by virtue of Theorem B, we get our conclusion.

Q.E.D.

Nom let $T^{0} M^{c}$ be a complexification of $T^{0} M$. Then we have $T^{0} M^{c}=$ $T^{0} M^{(1,0)} \oplus T^{0} M^{(0,1)}$ with respect to $\phi$, where

and

$$
T^{0} M^{(1,0)}=\left\{Z \in T^{0} M^{c}: \phi Z=\sqrt{-1} Z\right\}=\left\{X-\sqrt{-1} \phi X: X \in T^{0} M\right\}
$$

$$
T^{0} M^{(0,1)}=\left\{Z \in T^{0} M^{c}: \phi Z=-\sqrt{-1} Z\right\}=\left\{X+\sqrt{ }-1 \phi X: X \in T^{0} M\right\} .
$$

We are now in a position to prove the following

THEOREM 2. Let $M$ be a real hypersurface of $P_{n}(\boldsymbol{C})$. Then the following are equivalent:

(i) $M$ is locally equivalent to one of homogeneous real hypersurfaces of type $A_{1}$ and $A_{2}$.

(ii) $\xi$ is a frincipal curvature vector and $\nabla_{Z} \xi$ is a $(0,1)$-vector for any $Z \in$ $T^{0} M^{(0,1)}$.

Proof. For any $Z(=X+\sqrt{-1} \phi X) \in T^{0} M^{(0,1)}$, from $(1.5)$ we have 


$$
\nabla_{Z} \xi=\phi A X+\sqrt{-1} \phi A \phi X \in T^{0} M^{c}, \quad \text { where } X \in T^{0} M .
$$

(i) $\Rightarrow$ (ii): Since $\phi A=A \phi, \xi$ is a principal curvature vector. Then from (2.3) we get

$$
\begin{aligned}
\nabla_{z} \xi & =\phi A X+\sqrt{-1} \phi^{2} A X \\
& =\phi A X+\sqrt{-1}(-A X+\eta(A X) \xi) \quad(\text { from (1.3) }) \\
& =\phi A X-\sqrt{-1} A X .
\end{aligned}
$$

Then we find

$$
\begin{aligned}
\phi\left(\nabla_{z} \xi\right) & =\phi(\phi A X-\sqrt{-1} A X) \\
& =-A X+\eta(A X) \xi-\sqrt{-1} \phi A X \\
& =-\sqrt{-1}(\phi A X-\sqrt{-1} A X),
\end{aligned}
$$

which shows that $\nabla_{Z} \xi$ is a $(0,1)$-vector with respect to $\phi$.

(ii) $\Rightarrow(\mathrm{i}):$ From $(2.3)$ we have

$$
\phi\left(\nabla_{Z} \xi\right)=\phi(\phi A X+\sqrt{-1} \phi A \phi X)=-\sqrt{-1}(\phi A X+\sqrt{-1} \phi A \phi X) .
$$

This, together with (1.3), shows that

$$
\begin{aligned}
& -A X+\eta(A X) \xi+\sqrt{-1}(-A \phi X+\eta(A \phi X) \xi) \\
& =-\sqrt{-1} \phi A X+\phi A \phi X \text { for any } X(\perp \xi) .
\end{aligned}
$$

Since $\xi$ is a principal curvature vector, the equation (2.4) is reduced to $-A X$ $-\sqrt{-1} A \phi X=\phi A \phi X-\sqrt{ }-1 \phi A X$ for any $X(\perp \xi)$. Therefore we conclude that $\phi A=A \phi$.

Q.E.D.

REMARK 1. Let $M$ be a Kaehler manifold (with complex structure $J$ ). Then the following are equivalent:

(i) $L_{X} J=0$.

(ii) $\nabla_{Z} X$ is a $(0,1)$-vector for any $(0,1)$-vector $Z$.

Motivated by this fact, we established Theorem 2 .

Finally we prove the following

Proposition 1. Let $M$ be a real hypersurface of $P_{n}(\boldsymbol{C})$. Suppose that $\xi$ is a principal curvature vector and the corresponding principal curvature is non-zero. If $\nabla_{\xi} A=0$, then $M$ is locally congruent to one of homogeneous real hypersurfaces of type $A_{1}$ and $A_{2}$. 
Proof. By hypothesis we may put $A \xi=\alpha \xi$. Then from Proposition A, (1.3) and (1.5) we have

$$
\left(\nabla_{\xi} A\right) \xi=\nabla_{\xi}(A \xi)-A \nabla_{\xi} \xi=(\xi \alpha) \xi+\alpha \nabla_{\xi} \xi=0 .
$$

And hence " $\nabla_{\xi} A=0$ " implies

$$
\left.g\left(\left(\nabla_{\xi} A\right) X, Y\right)=0 \quad \text { (for any } X, Y \perp \xi\right) .
$$

On the other hand, for any $X\left(\in V_{r}=\{X: A X=r X, X \perp \xi\}\right)$ we get

$$
\begin{aligned}
g\left(\left(\nabla_{\xi} A\right) X, Y\right) & =g\left(\left(\nabla_{X} A\right) \xi+\phi X, Y\right) \quad(\text { from (1.7)) } \\
& =g\left(\nabla_{X}(A \xi)-A \nabla_{X} \xi+\phi X, Y\right) \\
& =g(\alpha \phi A X-A \phi A X+\phi X, Y) \quad \text { (from Proposition A and (1.5)) } \\
& =g(\alpha r \phi X-r A \phi X+\phi X, Y) \\
& =\left\{r\left(\alpha-\frac{\alpha r+2}{2 r-\alpha}\right)+1\right\} g(\phi X, Y) \quad \text { (from Proposition B) }
\end{aligned}
$$

Therefore the equation (2.5) asserts that

$$
r\left(\alpha-\frac{\alpha r+2}{2 r-\alpha}\right)+1=0 .
$$

Namely we find $\alpha\left(r^{2}-\alpha r-1\right)=0$. Since $\alpha \neq 0$, we have $r^{2}-\alpha r-1=0$ so that $r(2 r-\alpha)=\alpha r+2$, that is, $r=(\alpha r+2) /(2 r-\alpha)$. Therefore $\phi V_{r}=V_{r}$ so that our real hypersurface $M$ must be locally congruent to one of homogeneous ones of type $A_{1}$ and $A_{2}$ (cf. [8]). Of course a homogeneous real hypersurface of type $A_{1}$ and $A_{2}$ satisfies the condition " $\nabla_{\xi} A=0$ " (cf. Proposition C). Q. E. D.

REMARK 2. " $A \xi=0$ " implies " $\nabla_{\xi} A=0$ " (see the proof of Proposition 1).

REMARK 3. By an easy calculation we find the following:

$\nabla_{\xi} \xi=0$ (, that is, $\xi$ is principal) $\Leftrightarrow\left(\nabla_{\xi} \phi\right) X=0$ for any $X \in T M \Leftrightarrow\left(\nabla_{\xi} \phi\right)(\xi)=0$.

\section{Main results.}

To state our results, we prepare some fundamental equations of subbundles (cf. [6]). Let $F$ be a vector bundle over a Riemannian manifold $M$. Assume that $F$ has a metric connection. Then any subbundle $E$ of $F$ has an induced metric connection. Denote by $\nabla^{F}$ and $\nabla^{E}$ the connections of $F$ and $E$, respectively. Then we have

$$
\nabla_{X}^{F} v=\nabla_{X}^{E} v+A(X)(v) \quad \text { for any } v \in C^{\infty}(E) \text { and } X \in T M \text {, }
$$


where $A$ is a $\operatorname{Hom}\left(E, E^{\perp}\right)$-valued 1 -form on $M$ and $E^{\perp}$ is the orthogonal complement of $E$ in $F$ with respect to the metric on $F . A$ is called the second fundamental form of subbundle $E$ in $F . \quad E^{\perp}$ is also given a connection induced from $F$. Denote it by $\nabla^{E^{\perp}}$. Then we see that

$$
\nabla_{X}^{F} w=\nabla_{X}^{E^{\perp}} w+B(X)(w) \quad \text { for any } w \in C^{\infty}\left(E^{\perp}\right) \text { and } X \in T M,
$$

where $B$ is a $\operatorname{Hom}\left(E^{\perp}, E\right)$-valued 1 -form on $M$. It is easily seen that $A=-{ }^{t} B$, where ${ }^{t} B$ is the transpose of $B$ with respect to the metric on $F$.

Now let $M$ be a real hypersurface of $P_{n}(C)$. Then $T M$ is a subbundle of $T P_{n}(C)$ over $M$ and $T^{0} M=\{X \in T M: X \perp \xi\}$ is a subbundle of $T M$. Thus each of $T M$ and $T^{0} M$ has a metric connection induced from $T P_{n}(C)$. The orthogonal complement of $T^{0} M$ in $T P_{n}(\boldsymbol{C})$ with respect to the metric on $T P_{n}(\boldsymbol{C})$ is denoted by $N^{0} M$, which is also a subbundle of $T P_{n}(\boldsymbol{C})$ with the induced metric connection.

Denote by $\nabla^{0}$ and $\nabla^{\perp}$ the connections of $T^{0} M$ and $N^{0} M$, respectively. By (3.1) we have

$$
\begin{aligned}
& \nabla_{X} Y=\nabla_{X}^{0} Y+A_{1}(X)(Y) \\
& \tilde{\nabla}_{X} Y=\nabla_{X}^{0} Y+A_{2}(X)(Y) \quad \text { for any } Y \in C^{\infty}\left(T^{0} M\right) \text { and } X \in T M,
\end{aligned}
$$

where $A_{1}$ and $A_{2}$ are the second fundamental forms of the subbundle $T^{0} M$ in $T M$ and $T P_{n}(C)$, respectively. Note that the second fundamental form of $T M$ in $T P_{n}(C)$ coincides with the ordinary second fundamental form of the immersion $M \rightarrow P_{n}(\boldsymbol{C}) . \quad A_{2}$ is interpreted as a smooth section of $\operatorname{Hom}\left(T M, \operatorname{Hom}\left(T^{0} M, N^{0} M\right)\right)$. Set $A^{0}=\left.A_{2}\right|_{T^{0} M}$, which is a smooth section of $\operatorname{Hom}\left(T^{0} M, \operatorname{Hom}\left(T^{0} M, N^{0} M\right)\right)$. Note that any ruled real hypersurfaces in $P_{n}(C)$ may be characterized by the condition $A^{0} \equiv 0$. We here consider the covariant derivative of $A^{0}$ with respect to the connection on $\operatorname{Hom}\left(T^{0} M, \operatorname{Hom}\left(T^{0} M, N^{0} M\right)\right)$ induced from $T P_{n}(C)$. First of all we show the following fundamental relations.

PROPOSITION 2.

(i ) $A_{1}(X)(Y)=-g(\phi A X, Y) \xi$,

(ii) $A_{2}(X)(Y)=g(A X, Y) N-g(\phi A X, Y) \xi$,

(iii) $\nabla^{0} \phi=0$,

(iv) $\nabla_{X}^{\frac{1}{X}} \xi=g(A X, \xi) N$,

(v) $\nabla_{X}^{\perp} N=-g(A X, \xi) \xi$,

where $X \in T M$ and $Y \in C^{\infty}\left(T^{0} M\right)$. 
Proof. For any $X \in T M$ and $Y \in C^{\infty}\left(T^{0} M\right)$, we have

(i ) $g\left(A_{1}(X)(Y), \xi\right)=g\left(\nabla_{X} Y, \xi\right)=-g(Y, \phi A X)$,

(ii) $g\left(A_{2}(X)(Y), \xi\right)=G\left(\tilde{\nabla}_{X} Y, \xi\right)=g\left(\nabla_{X} Y, \xi\right)=-g(Y, \phi A X)$, $G\left(A_{2}(X)(Y), N\right)=G\left(\tilde{\nabla}_{X} Y, N\right)=g(A X, Y)$,

(iii) $\left(\nabla_{X}^{0} \phi\right)(Y)=\nabla_{X}^{0} \phi(Y)-\phi\left(\nabla_{X}^{0} Y\right)$

$$
\begin{aligned}
& =\nabla_{X} \phi(Y)-A_{1}(X)(\phi(Y))-\phi\left(\nabla_{X} Y-A_{1}(X)(Y)\right) \\
& =\left(\nabla_{X} \phi\right)(Y)+g(\phi A X, \phi Y) \xi \\
& =0
\end{aligned}
$$

where we have used $(1.1) \sim(1.5)$.

(iv) $\tilde{\nabla}_{X} \xi=\nabla_{X} \xi+g(A X, \xi) N=\phi A X+g(A X, \xi) N$,

which, together with (3.2), implies $\nabla_{\bar{X}}^{\frac{1}{X}} \xi=g(A X, \xi) N$.

(v) $\tilde{\nabla}_{\boldsymbol{X}} N=-A X$,

which, combined with (3.2), implies $\nabla_{X}^{\perp} N=-g(A X, \xi) \xi$.

Q.E.D.

The connection on $\operatorname{Hom}\left(T^{0} M, \operatorname{Hom}\left(T^{0} M, N^{0} M\right)\right)$ is also denoted by $\nabla^{0}$. The covariant derivative of $A^{0}$ is defined by

$$
\left(\nabla_{X}^{0} A^{0}\right)(Y)(Z)=\nabla_{X}^{\frac{1}{X}} A^{0}(Y)(Z)-A^{0}\left(\nabla_{X}^{0} Y\right)(Z)-A^{0}(Y)\left(\nabla_{X}^{0} Z\right)
$$

for any $X \in T M$ and $Y, Z \in C^{\infty}\left(T^{0} M\right)$.

Now we prove

Proposition 3. For any $X \in T M$ and $Y, Z \in C^{\infty}\left(T^{0} M\right)$,

$$
\left(\nabla_{X}^{0} A^{0}\right)(Y)(Z)=\Psi(X, Y, Z) N+\Psi(X, Y, \phi Z) \xi,
$$

where $\Psi$ is the trilinear tensor defined by

$$
\begin{aligned}
\Psi(X, Y, Z) & =g\left(\left(\nabla_{X} A\right)(Y), Z\right)-\eta(A X) g(\phi A Y, Z) \\
& -\eta(A Y) g(\phi A X, Z)-\eta(A Z) g(\phi A X, Y) .
\end{aligned}
$$

Proof. We have from Proposition 2

$$
\begin{aligned}
\left(\nabla_{X}^{0} A^{0}\right)(Y)(Z)= & \nabla_{\dot{X}}^{1} A^{0}(Y)(Z)-A^{0}\left(\nabla_{X}^{0} Y\right)(Z)-A^{0}(Y)\left(\nabla_{X}^{0} Z\right) \\
= & \left\{g\left(\nabla_{X}(A Y), Z\right)+g\left(A Y, \nabla_{X} Z\right)\right\} N-\eta(A X) g(A Y, Z) \xi \\
& -\left\{g\left(\nabla_{X}(\phi A Y), Z\right)+g\left(\phi A Y, \nabla_{X} Z\right)\right\} \xi-\eta(A X) g(\phi A Y, Z) N
\end{aligned}
$$




$$
\begin{aligned}
&-g\left(A\left(\nabla_{X}^{0} Y\right), Z\right) N+g\left(\phi A\left(\nabla_{X}^{0} Y\right), Z\right) \xi-g\left(A Y, \nabla_{X}^{0} Z\right) N \\
&+g\left(\phi A Y, \nabla_{X}^{0} Z\right) \xi \\
&=\left\{g\left(\left(\nabla_{X} A\right)(Y), Z\right)-\eta(A Y) g(\phi A X, Z)-\eta(A X) g(\phi A Y, Z)\right. \\
&-\eta(A Z) g(\phi A X, Y)\} N+\{-\eta(A X) g(A Y, Z)-\eta(A Y) g(A X, Z) \\
&\left.-g\left(\phi\left(\nabla_{X}(A Y)\right), Z\right)+g\left(\phi A\left(\nabla_{X} Y\right), Z\right)-\eta(A \phi Z) g(\phi A X, Y)\right\} \xi, \\
& \text { Q. E. D. }
\end{aligned}
$$

which implies (3.6).

Recall the definition of $\eta$-parallelity of $A$. We say that $A^{0}$ is $\eta$-parallel if $\nabla_{X}^{0} A^{0} \equiv 0$ for any $X \in C^{\infty}\left(T^{0} M\right)$.

The main purpose of this paper is to prove the following

THEOREM 3. Let $M$ be a real hypersurface of $P_{n}(C)$. Assume that $A^{0}$ is $\eta$ parallel. Then $M$ is locally congruent to one of the following:

(1) a homogeneous real hypersurface of type $A_{1}$,

(2) a homogeneous real hypersurface of type $A_{2}$,

(3) a homogeneous real hypersurface of type $B$,

(4) a real hypersurface in which $T^{0} M$ is integrable and its integral manifold is a totally geodesic $P_{n-1}(\boldsymbol{C})$ (, that is, $M$ is a ruled real hypersurface),

(5) a real hypersurface in which $T^{0} M$ is integrable and its integral manifold is a complex quadric $\boldsymbol{Q}_{n-1}$.

Proof. By Proposition 3, $A^{0}$ is $\eta$-parallel if and only if $\Psi(X, Y, Z)=0$ for any $X, Y, Z \in C^{\infty}\left(T^{0} M\right)$, that is,

$$
\begin{aligned}
g\left(\left(\nabla_{X} A\right)(Y), Z\right)= & \eta(A X) g(\phi A Y, Z)+\eta(A Y) g(\phi A X, Z) \\
& +\eta(A Z) g(\phi A X, Y) \quad \text { for any } X, Y, Z \in C^{\infty}\left(T^{0} M\right)
\end{aligned}
$$

Therefore we must study real hypersurfaces (in $P_{n}(C)$ ) which satisfy the equation (3.8). Since the Codazzi equation (1.7) tells us that $g\left(\left(\nabla_{X} A\right) Y, Z\right)$ is symmetric for any $X, Y$ and $Z\left(\in T^{0} M\right)$, exchanging $X$ and $Y$ in (3.8), we obtain $g(Y, \phi A X) \eta(A Z)=g(X, \phi A Y) \eta(A Z)$ so that

$$
\eta(A Z) g((A \phi+\phi A) X, Y)=0 \quad \text { for any } X, Y, Z\left(\in T^{0} M\right) .
$$

Now we assume that $\eta(A Z)=0$ for any $Z\left(\in T^{0} M\right)$, that is, $\xi$ is a principal curvature vector. Then the equation (3.8) shows that $g\left(\left(\nabla_{X} A\right) Y, Z\right)=0$ for any $X, Y, Z\left(\in T^{0} M\right)$, that is, the second fundamental form $A$ of $M$ is $\eta$-parallel. And hence our real hypersurface $M$ is locally congruent to one of homogeneous ones of type $A_{1}, A_{2}$ and $B$ (cf. Theorem C). Next we assume that $\xi$ is not a 
principal curvature vector. Then the equation (3.9) tells us that the holomorphic distribution $T^{0} M$ is integrable (cf. Proposition D). Of course the integral manifold $M^{0}$ of $T^{0} M$ is a complex hypersurface (with complex structure $\phi$ ) in $P_{n}(\boldsymbol{C})$. Moreover, the second fundamental form $A^{0}$ of $M^{0}$ is parallel (, which is equivalent to (3.8)). Therefore we conclude that $M^{0}$ is locally congruent to $P_{n-1}(\boldsymbol{C})$ or $\boldsymbol{Q}_{n-1}$ (cf. [10]).

Q.E.D.

As an immediate consequence of Theorem $C$ and (3.8), we get

THEOREM 4. Let $M$ be a real hypersurface of $P_{n}(C)$. Then $A^{0}$ is $\eta$-parallel and $\xi$ is a principal curvature vector if and only if $M$ is locally congruent to one of homogeneous real hypersurfaces of type $A_{1}, A_{2}$ and $B$.

In addition, from Theorem $\mathrm{C}$, Theorem $\mathrm{D}$ and Theorem 3 , we find

THEOREM 5. Let $M$ be a real hypersurface of $P_{n}(C)$. Then $A^{0}$ is $\eta$-parallel and the second fundamental form of $M$ is $\eta$-parallel if and only if $M$ is locally congruent to one of homogeneous real hypersurfaces of type $A_{1}, A_{2}$ and $B$ or $a$ ruled real hypersurface.

REMARK 4. We now denote by $H$ the sectional curvature of a holomorphic 2-plane (with respect to $\phi$ ) on a real hypersurface $M$. Kimura ([4]) determined real hypersurfaces (in $P_{n}(\boldsymbol{C})$ ) on which $H$ is constant. He showed the following

THEOREM E ([4]). Let $M$ be a real hypersurface of $P_{n}(C)(n \geqq 3)$ on which $H$ is constant. Then $M$ is one of the following:

(a) a homogeneous real hypersurface of type $A_{1}(H>4)$,

(b) a real hypersurface in which $T^{0} M$ is integrable and its integral manifold is a totally geodesic $P_{n-1}(C)$ (, that is, $M$ is a ruled real hypersurface) $(H=4)$,

(c) a real hypersurface in which there is a foliation contained in some complex hyperplane $P_{n-1}(C)$ as a ruled real hypersurface $(H=4)$.

Our aim here is to give a characterization of the cases (b), (c) in Theorem E. We prove

Proposition 4. Let $M$ be a real hypersurface of $P_{n}(\boldsymbol{C})(n \geqq 3)$. If $T^{0} M$ is a curvature invariant subspace of $T M$ and $\xi$ is not a principal curvature vector, then $M$ is locally congruent to one of the cases (b), (c) in Theorem E.

Proof. Since $R\left(T^{0} M, T^{0} M\right) T^{0} M \subset T^{0} M$, the equation (1.6) yields 


$$
\begin{aligned}
0 & =g(R(X, Y) Z, \xi) \\
& =g(A Y, Z) g(A X, \xi)-g(A X, Z) g(A Y, \xi)
\end{aligned}
$$

for any $X, Y, Z \in T^{0} M$ and $\xi=-J N$.

Then we have

$$
\eta(A X) \phi A Y=\eta(A Y) \phi A X \quad \text { for any } X, Y \in T^{0} M .
$$

We here consider a linear transformation $\phi A: T^{0} M \rightarrow T^{0} M$. Note that

$$
\operatorname{rank}(\phi A) \leqq 1 \quad \text { at each point of } M \text {. }
$$

Suppose that $\operatorname{rank}(\phi A) \geqq 2$ at a certain point $x$ of $M$. Then there exist $X, Y \in T_{x}^{0} M$ such that

$$
\phi A X \neq 0, \quad \phi A Y \neq 0 \text { and } g(\phi A X, \phi A Y)=0 .
$$

So from (3.10) and (3.12) we see

$$
\eta(A X)=0 \text {. }
$$

It follows from (3.10) and (3.13) that

$$
\eta(A Y)=0 \quad \text { for any } Y(\perp X) .
$$

Therefore, from (3.13) and (3.14) we find that $\xi$ is a principal curvature vector at $x$, which is a contradiction.

Then (3.11) asserts that the Gauss equation (1.6) is reduced to

$$
\begin{aligned}
g(R(X, Y) Z, W)= & g(Y, Z) g(X, W)-g(X, Z) g(Y, W)+g(\phi Y, Z) g(\phi X, W) \\
& -g(\phi X, Z) g(\phi Y, W)-2 g(\phi X, Y) g(\phi Z, W),
\end{aligned}
$$

that is,

$$
\begin{aligned}
R(X, Y) Z= & g(Y, Z) X-g(X, Z) Y+g(\phi Y, Z) \phi X-g(\phi X, Z) \phi Y \\
& -2 g(\phi X, Y) \phi Z \quad \text { for any } X, Y, Z \in T^{0} M .
\end{aligned}
$$

Then we conclude that our real hypersurface $M$ satisfies that $H=4$. Therefore Theorem $\mathrm{E}$ tells us that $M$ is locally congruent to one of the cases (b), (c). Of course the cases (b), (c) satisfy the hypothesis of Proposition 4. Q. E. D.

We here provide a geometric meaning of the condition "the second fundamental form of $M$ is $\eta$-parallel". The following is due to Nakagawa.

PROPOSITION 5. Let $M$ be a real hypersurface of $P_{n}(C)$. Then the following are equivalent :

(i) The second fundamental form of $M$ is $\eta$-parallel. 
(ii) Every geodesic $\gamma=\gamma(t)(t \in I)$ of $M$ such that $\gamma^{\prime}(t)$ is orthogonal to $\xi$ (for any $t \in I)$, considered as a curve in $P_{n}(\boldsymbol{C})$, has constant first curvature along $\gamma$.

Proof. We find that the condition (ii) is equivalent to $g\left(\left(\nabla_{X} A\right) X, X\right)=0$ for any $X\left(\in T^{0} M\right)$. On the other hand, the Codazzi equation shows that $g\left(\left(\nabla_{X} A\right) Y, Z\right)$ is symmetric for any $X, Y$ and $Z\left(\in T^{0} M\right)$. And hence the con$\begin{array}{ll}\text { dition (i) is equivalent to the condition (ii). } & \text { Q.E.D. }\end{array}$

REMARK 5. The first author ([8]) proved the following:

Let $M$ be a real hypersurface of $P_{n}(\boldsymbol{C})$. Then every geodesic $\gamma$ of $M$, considered as a curve in $P_{n}(\boldsymbol{C})$, has constant first curvature along $\gamma$ if and only if $M$ is locally congruent to one of homogeneous real hypersurfaces of type $A_{1}$ and $A_{2}$.

REMARK 6. The authors do not know how to construct a real hypersurface $M$ with $M^{0}=\boldsymbol{Q}_{n-1}$ (, that is, $M$ is of case (5) in Theorem 3).

\section{References}

[1] Cecil, T.E. and Ryan, P. J., Focal sets and real hypersurfaces in complex projective space, Trans. Amer. Math. Soc. 269 (1982), 481-499.

[2] Ki, U.H., Nakagawa, H. and Suh, Y.J., Real hypersurfaces with harmonic Weyl tensor of a complex space form, a preprint.

[3] Kimura, M., Real hypersurfaces and complex submanifolds in complex projective space, Trans. Amer. Math. Soc. 296 (1986), 137-149.

[4] Kimura, M., Sectional curvatures of holomorphic planes on a real hypersurface in $P^{n}(C)$, Math. Ann. 276 (1987), 487-497.

[5] Kimura, M. and S. Maeda, On real hypersurfaces of a complex projective space, Math. Z. 202 (1989), 299-311.

[6] Kobayashi, S., Differential geometry of complex vector bundles, Publ. Math. Soc. Japan 15, Iwanami Shoten, Publ. and Princeton Univ. Press, 1987.

[7] M. Kon, Pseudo-Einstein real hypersufaces in complex space form, J. Diff. Geom. 14 (1979), 339-354.

[8] Maeda, S., Real hypersurfaces of complex projective spaces, Math. Ann. 263 (1983), 473-478.

[9] Maeda, Y., On real hypersurfaces of a complex projective space, J. Math. Soc. Japan 28 (1976), 529-540.

[10] Nakagawa, H. and Takagi, R., On locally symmetric Kaehler submanifolds in a complex projective space, J. Math. Soc. Japan 28 (1976), 638-667.

[11] Okumura, M., On some real hypersurfaces of a complex projective space, Trans. Amer. Math. Soc. 212 (1975), 355-364.

[12] Okumura, Compact real hypersurfaces of a complex projective space, J. Diff. Geom. 12 (1977), 595-598.

[13] Takagi, R., On homogeneous real hypersurfaces in a complex projective space, 
Osaka, J. Math. 10 (1973), 495-506.

[14] Takagi, R., Real hypersurfaces in a complex projective space with constant principal curvatures I, II, J. Math. Soc. Japan 27 (1975), 43-53, 507-516.

[15] Udagawa, S., Bi-order real hypersurfaces in a complex projective space, Kodai Math. J. 10 (1987), 182-196.

Sadahiro Maeda

Department of Mathematics

Kumamoto Institute of Technology

Ikeda $4-22-1$

Kumamoto 860, Japan

Seiichi Udagawa

Department of Mathematics

School of Medicine

Nihon University

Itabashi, Tokyo 173, Japan 